\section{OPEN}

SUBJECT AREAS:

TISSUE ENGINEERING

EXTRACELLULAR MATRIX

Received

25 September 2014

Accepted

12 December 2014

Published

22 January 2015

Correspondence and requests for materials should be addressed to B.R.-R. (barbara. rothen@unifr.ch)

* Current address: Agroscope, Institute for Livestock Sciences ILS, Rte de la Tioleyre 4, CP 64, $\mathrm{CH}-1725$ Posieux, Switzerland.

\title{
Engineering an in vitro air-blood barrier by 3D bioprinting
}

\author{
Lenke Horváth' ', Yuki Umehara', Corinne Jud' *, Fabian Blank², Alke Petri-Fink' \\ \& Barbara Rothen-Rutishauser ${ }^{1}$
}

\author{
${ }^{1}$ Adolphe Merkle Institute, University of Fribourg, Chemin des Verdiers 4, CH-1700 Fribourg, Switzerland, ${ }^{2}$ Department of \\ Respiratory Medicine, Bern University Hospital, CH-3010 Bern, Switzerland.
}

Intensive efforts in recent years to develop and commercialize in vitro alternatives in the field of risk assessment have yielded new promising two- and three dimensional (3D) cell culture models. Nevertheless, a realistic 3D in vitro alveolar model is not available yet. Here we report on the biofabrication of the human air-blood tissue barrier analogue composed of an endothelial cell, basement membrane and epithelial cell layer by using a bioprinting technology. In contrary to the manual method, we demonstrate that this technique enables automatized and reproducible creation of thinner and more homogeneous cell layers, which is required for an optimal air-blood tissue barrier. This bioprinting platform will offer an excellent tool to engineer an advanced 3D lung model for high-throughput screening for safety assessment and drug efficacy testing.

$\mathrm{n}$ the field of regulatory toxicity animal testing is still the most prevalent model used for risk assessment, study of diseases and new drug development ${ }^{1}$. However, three main factors create a strong need for in vitro alternatives $^{2,3}$ : Regulatory pressure to ban animal testing ${ }^{4}$, pressure to reduce cost in drug development ${ }^{5}$, and concerns with respect to the significance of animal experiments to model human health ${ }^{6}$. Therefore, new concepts for more efficient, cheaper and evidence-based test strategies were proposed such as cell culture models which provide the possibility to investigate processes such as toxic effects on human cells, which cannot be conducted in vivo where mainly rodents are used. Besides having the potential to replace traditional animal experiments, in vitro models represent a sophisticated and reproducible system that allows us to deconstruct and manipulate complex cellular pathways ${ }^{7}$, hence they can be used as a first step towards understanding how an agent will react in the body. The lung is the main portal of entry for aerosols (reviewed in ${ }^{8}$ ) and despite the existence of epithelial tissue barriers, respiratory diseases are frequent and increasing ${ }^{9,10}$. Recently, more attention has been directed towards elucidating how and when the antigens evade these barriers and therefore many alternative cell models have been established ${ }^{11}$.

Respiratory tissue engineering has advanced significantly within the last years and several approaches are described in the literature that can be used to mimic the human pulmonary epithelial tissue barrier by applying $2 \mathrm{D}$ and 3D models ranging from simple mono-cultures ${ }^{12,13}$ to highly sophisticated 3D constructs including cocultures of various cells and/or scaffold supports ${ }^{14-17} .3 \mathrm{D}$ cell cultures represent advanced, more complex systems since they involve cell-cell interactions mimicking the native tissue and its microenvironment more realistically ${ }^{17}$. In the last decade, in vitro cell culture models that closely mimic the conducting airways as the trachea and bronchi's, were introduced on the market (e.g. products of Epithelix SàrL, CellnTec GmbH). Nevertheless, a huge gap remains in recreating in vitro alternatives that realistically mimic the human air-blood tissue barrier structure. In addition, a great drawback of the current co-culture models is the use of commercially available two chamber systems, as well as culturing different cell types on two sides of a relatively thick (about $10 \mu \mathrm{m}$ ) porous membrane composed of materials such as for example poly-ethylene terephthalate (PET). This is not optimal for the development of an air-blood barrier model where the real barrier corresponds merely to two very thin cytoplasmic lamellae of the endothelial and epithelial cells separated by a single basement membrane (BM); the average overall thickness of this tissue barrier amounts to about $1.6 \mu \mathrm{m}^{18,19}$.

Significant progress in microfluidic technology has demonstrated a great potential for developing advanced cellular and tissue structures with high physiological relevance, such as the biomimetic microdevices also called "organs-on-a-chip", which will be extremely useful systems for testing new drug candidates or risk assessment of engineered nanomaterials ${ }^{20-23}$. The introduction of cyclical mechanical strain simulating breathing conditions in the lung also enabled a huge step forward in recreating realistic models of the respiratory tissue ${ }^{20,21,24}$. The lung on 

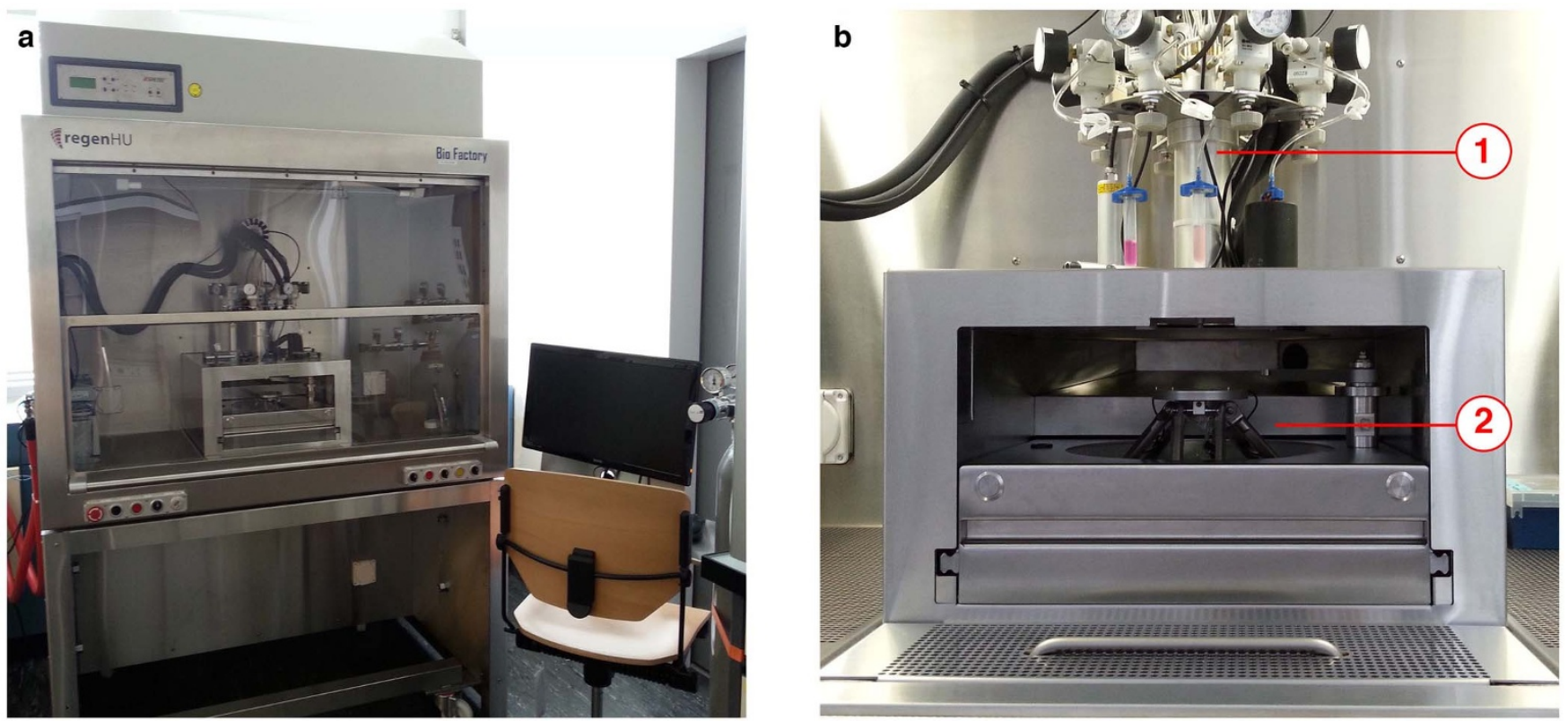

c

$1^{\text {st }}$ printed layer

$2^{\text {nd }}$ printed layer

$3^{\text {rd }}$ printed layer

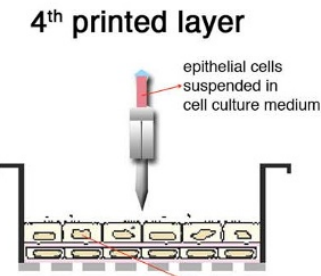

Engineered two-cell layer barrier system

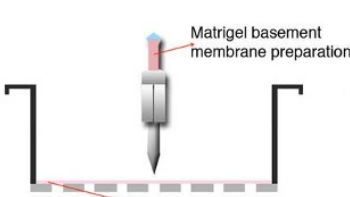

Matrigel ${ }^{\mathrm{TM}} \mathrm{I}$.

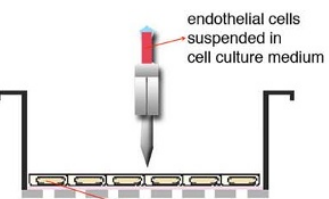

EA.hy926

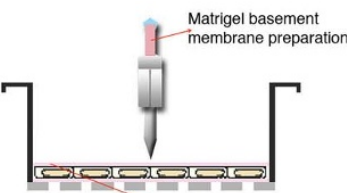

Matrigel $^{\mathrm{TM}}$ II.

A549

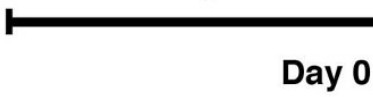

Day 2

Day 5

Figure 1 Bioprinting system, fabrication of 3D constructs and schematic illustration of the cell patterning timeline process. (a) The BioFactory ${ }^{\circledR}$ is a bioprinter developed for creating 3D organotypic structures for tissue engineering and drug discovery. The main component of the instrument is (b) the process unit comprising the tool changer (1) with 3 workstations equipped with print heads and the building cell area with the building platform (2). (c) Schematic of the timeline for bioprinting the two cell-layer barrier system. On day 0, a thin layer of extracellular matrix (ECM) is printed on porous membranes, and subsequently a layer of EA.hy926 endothelial cells is printed on Matrigel ${ }^{\mathrm{TM}}$. On day 2, a second ECM layer is printed on endothelial cells to ensure the adhesion of the next printed layer of A549 epithelial cells. The cells are cultivated for additional 3 days before their 3D organization, viability, proliferation and barrier quality are assessed. The manual co-culture assembly follows the timeline described for bioprinting including the same steps however made manually, i.e. manually pipetting the ECM/cell layers.

a chip platform described by Huh et al. ${ }^{20}$ combined alveolar epithelial and microvascular endothelial cells in two closely apposed microchannels separated by a $10 \mu \mathrm{m}$ porous, flexible membrane to mimic the architecture of the air-capillary barrier. The cyclic stretching of the interface simulating breathing motions was introduced by vacuum chambers on the side. This microsystem was later used to model drug toxicity induced pulmonary edema ${ }^{21}$. Recently, another system, i.e. the captive bubble surfactometer, was introduced allowing to investigate lung cell structure and function in vitro under breathing conditions ${ }^{24}$.

A different approach in tissue engineering strategies has enabled the emergence of bioprinting technology employing cells and bioactive matrix components ${ }^{25,26}$ for design and biofabrication of in vitro physiological models that is expected to tremendously impact our understanding of physiological mechanisms, disease pathologies and significantly enhance drug development. The great power of bioprinting technology is to (i) provide standardized and reproducible cell models for high-throughput screening, (ii) customization of specific (diseased) tissue models, and (iii) the possibility to spatially and temporally control the deposition of biomaterials to generate complex 3D constructs in a layer-by-layer fashion ${ }^{27,28}$. Along with the development of various bioprinting strategies (reviewed $i^{27,29-32}$ ) there has been increasing interest in implementing these for applications in regenerative medicine, cancer research, but also for use in drug discovery, toxicology or basic research ${ }^{30}$. Until now 3D bioprinting methods have been used to create multiple tissue types, including $\operatorname{cardiac}^{33-36}$, bone and cartilage tissue ${ }^{36-40}$, vascular networks ${ }^{41-43}$, multi-layered skin $^{44}$ and nerve grafts ${ }^{45,46}$. Cell printing platforms were applied to construct $3 \mathrm{D}$ tumor models ${ }^{47,48}$ to $\mathrm{mimic} /$ simulate the physiological tumor microenvironment. Other studies reported about the development of bioprinting processes to create liver micro-organ for examining in vitro drug metabolism and pharmokinetics ${ }^{49,50}$ and to deposit human embryonic stem cells directly on microwell plates, resulting formation of spheroid aggregates ${ }^{51}$. These promising examples employing cell bioprinting could pave the way for establishing more accurate models for in vitro drug development and toxicity testing.

To best of our knowledge no work so far has been reported about lung tissue engineering using 3D bioprinting. The scope of our work was to develop a complex, yet reproducible and fully automatized, engineered 3D air-blood tissue barrier using an innovative valve-based bioprinting approach. An existing bioprinter, the BioFactory ${ }^{\circledR}$ (Fig. 1a-b; regenHU Ltd., Switzerland), has been adapted to engineer 

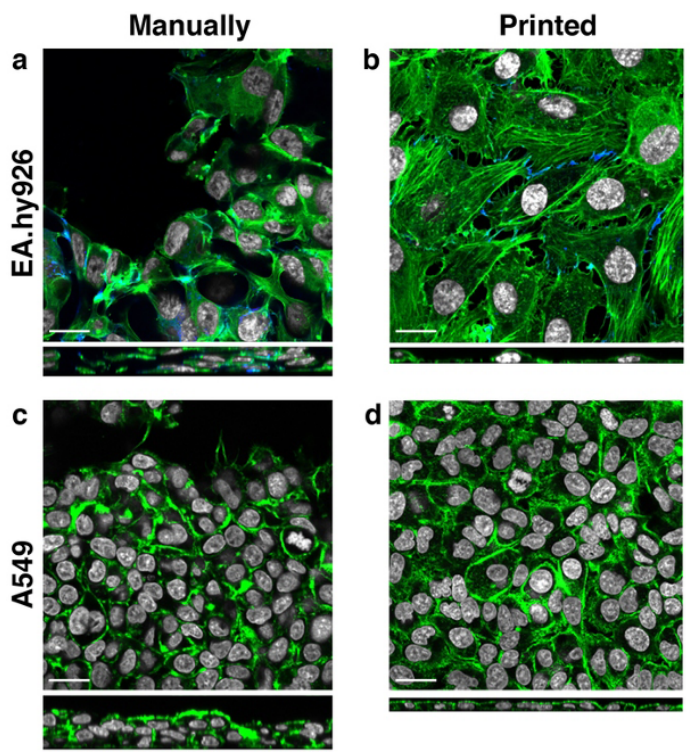

Figure $2 \mid$ Characterization and comparison of manually seeded and bioprinted mono-cultures by LSM. Immunofluorescence labelling of Factin cytoskeleton $(\square)$ and nuclei $(\square)$ in (a,b) EA.hy926 and (c,d) A549 mono-cultures cultivated for 3 days following $(\mathrm{a}, \mathrm{c})$ manual seeding and $(\mathrm{b}, \mathrm{d})$ bio-printing on Matrigel ${ }^{\mathrm{TM}} \mathrm{BM}$. Endothelial cells were labelled in addition with specific cell adhesion marker PECAM-1 (platelet endothelial adhesion molecule also referred to as CD31 ( $\square$ )). Single optical sections are presented in the $x-y$ projection (central image) with respective side views in $\mathrm{xz}$ (bottom). Scale bars correspond to $20 \mu \mathrm{m}$.

a human air-blood barrier analogue consisting of A549 (alveolar epithelial type II cells; AT-II), EA.hy926 (endothelial) cells separated by thin BM Matrigel ${ }^{\mathrm{TM}}$ by applying a layer-by-layer printing approach (Fig. 1c) in order to optimize cell culture conditions in regards to the structure and function of the human organ. Initial experiments have been performed using epithelial and endothelial mono-cultures, which were then further assembled into co-cultures and the functional-structural relation of the tissue constructs was examined by various stateof-the art methods. In addition, we report on the comparison between two approaches for $3 \mathrm{D}$ tissue modelling, i.e. conventional manual versus bioprinting.

\section{Results}

Comparison of cellular morphology and cell-cell contacts in manually seeded and bioprinted cultures. To study the $3 \mathrm{D}$ structure of cells printed on Matrigel ${ }^{\mathrm{TM}}$ in single and co-cultures and to compare it with the conventional manual method, cells were fixed after three days in culture and investigated with laser scanning microscopy (LSM). According to the confocal micrographs obtained following immunostaining of the cells for F-actin (green) and nuclei (white) it could be shown that both, endothelial (Fig. 2a-b) and epithelial cells (Fig. 2c-d) exhibited different morphology depending on the seeding method, i.e., manual (Fig. 2a, c) vs. printing (Fig. 2b, d). Cells seeded by conventional manual method typically grew in patches (Fig. 2a, c) often overgrowing each other forming multi-layered clusters (side views of Fig. 2a, c), although the cells were also seeded as single cells and not in clumps as shown by phase contrast pictures (Supplementary Fig. S1). In contrast, the corresponding printed cells (Fig. 2b, d) spread over the available growth surface to form confluent thin monolayers (side views of Fig. 2b, d). A549 cells had typical polarized, cuboidal morphology ${ }^{52}$, while EA.hy926 cells formed simple flat sheets covering larger growth surfaces. The bioprinting process was fine-tuned in order to achieve very thin layers of Matrigel $^{\mathrm{TM}}$ that induced a more optimal growth of the epithelial and endothelial cells, i.e. they formed thin cell layers as mentioned above.
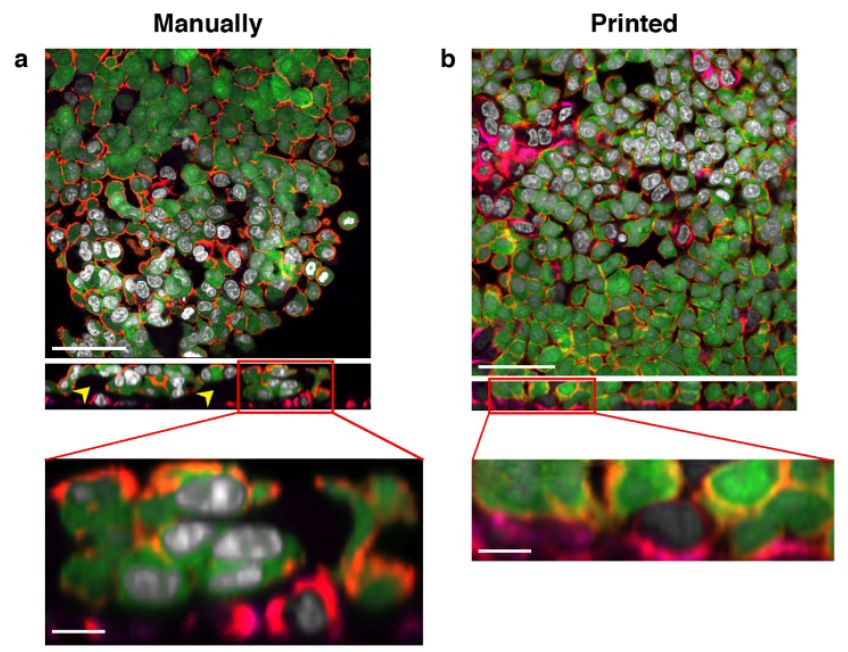

c

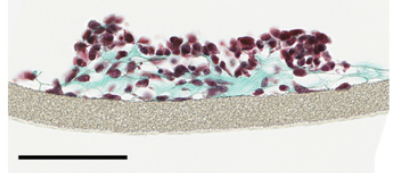

d

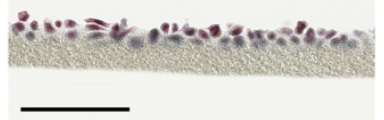

Figure 3 Characterization and comparison of manually seeded and bioprinted co-cultures by LSM and brightfield microscopy. Laser scanning micrographs of two cell-layers composed of first layer of Matrigel ${ }^{\mathrm{TM}} \mathrm{BM}$, endothelial cells, second Matrigel ${ }^{\mathrm{TM}}$ layer, and epithelial cells by (a) manual and (b) bioprinting approaches after 3 days. All cells were stained for F-actin (red) and nuclei (white). A549 cells are represented in green, endothelial cells were labelled with VE-cadherin (pink). Note the formation of cell multilayers and the presence of thick Matrigel ${ }^{\mathrm{TM}}$ layer (yellow arrowheads) under manual condition. Images are presented in xy projections with representative side views. Parts of side views are magnified for better visualization of cell-cell contacts. Brightfield micrographs of (c) conventionally seeded and (d) bioprinted paraffin embedded histological cross sections stained with Masson-Goldner trichrome coloration. Cytoplasm is stained red, collagen fibers of the ECM Matrigel ${ }^{\mathrm{TM}}$ green and cell nuclei dark brown. Note the thick ECM layers in c) which are hardly visible in d). Scale bars are (a-b) $50 \mu \mathrm{m}$, (a-b close-up) $10 \mu \mathrm{m}$ and (c-d) $100 \mu \mathrm{m}$.

Similar results were observed for the two cell-layers of EA.hy926 and A549 cells (Fig. 3). For these experiments we have used A549 cells labelled with green fluorescent protein (GFP) to distinguish them from the endothelial cells. The GFP-A549 cells grew similar to non-labelled cells (data not shown). The manual seeding approach often produced in addition to discrete multi-layered clusters an apparent thick ECM layer between endothelial and epithelial cells (Fig. 3a, yellow arrowheads pointing to dark regions designating the basement membrane and Fig. 3a close-up) resulting often in a lack of direct cell-cell contacts/communication of different cell types. On the other hand, printed cell layers are structurally in close proximity to each other (Fig. 3b close-up), ensuring complex cell-cell interactions, hence having higher structural and functional resemblance of complex cell networks found at the air-blood barrier in vivo. Due to the fact that the BM layers were not visible by LSM, we investigated the two cell-layers by brightfield microscopy following Masson Goldner trichrome staining of paraffin embedded histological sections (Fig. 3c, d). This staining method is recommended for distinguishing cells from surrounding connective tissue, specifically collagen. Representative brightfield micrographs of stained manual (Fig. 3c) and printed co-cultures (Fig. 3d) corroborate our previous observations with LSM, i.e. presence of thick BM layers by manual approach (Fig. $3 c$ green staining) and thin printed layers that are hard to detect in the samples even with the employed histological staining. 
Assessment of cell growth, viability and integrity of manually seeded and bioprinted cultures. A phase-contrast microscope integrated in a tissue culture incubator (Nikon Biostation CT; Nikon Instruments, Zurich, Switzerland) was used to observe the growth of endothelial-, epithelial mono-cultures and the cocultures over a 3 day experimental period (Fig. $4 a-f$ ). Compared to the conventional manual seeding where cells grow typically in discrete spheroid-like clusters, which can be observed already $2 \mathrm{~h}$ after seeding (Supplementary Fig. S1, Fig. 4a, c and Supplementary Movies S1, S3), both, printed EA.hy926 (Supplementary Fig. S1, Fig. $4 \mathrm{~b}$ and Supplementary Movie S2) and A549 cells (Supplementary Fig. S1, Fig. 4d and Supplementary Movie S4) show homogeneous distribution and exponential growth over the surface of membranes and reach confluence on the $3^{\text {rd }}$ day of incubation. Figures $4 \mathrm{e}$ and $4 \mathrm{f}$ display merged phase contrast and fluorescence micrographs of co-cultures assembled by manual (Fig. 4e) and printing (Fig. 4f) approach (see also Supplementary Movies S5 and S6). Endothelial (phase contrast) and epithelial cells (designated green fluorescent on same micrographs) exhibited similar growth to the respective mono-culture conditions, i.e., heterogeneous discrete clusters under manually seeded conditions and homogeneous cell distribution when the bioprinting approach was employed. At the last time point (Fig. 4e, f: 72 h), cells reached near-confluence state in both conditions (A549 cells are visible only).

The cell viability was assessed by Trypan Blue exclusion assay after $24 \mathrm{~h}$ and by measuring the activity of lactate dehydrogenase (LDH) released into the cell culture medium of samples after 3 days yielded by the conventional manual and the novel bioprinting approach. Manually seeded cells being in culture for $24 \mathrm{~h}$ displayed high viability with dead cells occurring only occasionally (Supplementary Fig. S2). Printed epithelial cells showed higher viability than printed endothelial cells as displayed on representative micrographs of Supplementary Figure 2. Cells incubated in $70 \%$ ethanol for 30 min served as positive controls resulting in $100 \%$ blue labelled cells. For LDH release measurements, cells lysed with Triton X-100 (treated for $24 \mathrm{~h}$ prior to the endpoint) revealing the maximum $\mathrm{LDH}$ release were considered as the reference point (positive control; value $=100 \%$ ) that is indicated as dashed red line on the plot (Fig. 4g). The viability of the endothelial and epithelial mono-cultures or co-cultures was essentially similar regardless of the approach (manual vs. printing) used. The percentage of dead A549 cells and epithelialendothelial co-cultures was similar and below 5\%, whether the cells were seeded by manual or printing method yielding an overall viability of $>95 \%$ for these cultures. Endothelial cells were found to be more sensitive with $14.2 \% \pm 4.5 \%$ dead cells after bioprinting and $10.4 \% \pm 5.4 \%$ dead cells following manual seeding resulting in about $\geq 86 \%$ cell survival on the $3^{\text {rd }}$ day of cultivation.

The barrier quality (i.e. tightness) of A549 and EA.hy926 cells in mono- and co-culture was investigated by measuring the translocation of Blue Dextran molecules from the apical to the basolateral compartment of the inserts after 3 days of cell growth. The overall leakage of Blue Dextran in an empty transwell insert without cells was used as the reference point (negative control; value $=1$ ) and is indicated as dashed red line on the respective plot (Fig. 4h). Printed mono-cultures of the two cell types showed higher tightness than the corresponding manual samples with epithelial cells showing significantly higher barrier qualities as compared to endothelial cells (both manual and printed). Printed endothelial cells were significantly less permeable than manually seeded cells while no significant difference was found between printed vs. manual epithelial cells. On the other hand, the manually assembled co-culture of EA.hy926 and A549 cells allowed significantly lower translocation of Blue Dextran than in the case of printed sample. This phenomenon, i.e. seemingly tighter barrier in manual co-cultures can be explained by the presence of manually dispersed thick layers of ECM in contrast to thin ECM layers achieved by printing. Namely, we have observed that ECM thickness influences the translocation of Blue Dextran under acellular conditions (Supplementary Fig. S3) insinuating that manual and printed co-cultures have similar barrier qualities.

\section{Discussion}

In this study we demonstrate for the first time, the engineering of a lung tissue analogue closely recapitulating the in vivo human airblood barrier architecture with a highly precise, reproducible biomanufacturing technique. The applied prototyping technology was a valve-based bioprinting process for layer-by-layer fabrication of 3D tissue constructs. Initial experimentation for testing the positional repeatability and accuracy of the printer showed that this technology enables highly precise and reproducible patterning in a controlled spatial arrangement (Supplementary Fig. S4). The printing process was optimized to operate under cell friendly low pressure conditions to deposit multiple cell types and ECM.

The two cell-layer model was designed in order to achieve a close physiological resemblance to the microenvironment of the native tissue, where the epithelial cell layer is only separated by a thin BM from the underlying endothelium. Similarly, we employed Matrigel $^{\mathrm{TM}} \mathrm{BM}$ extract that corresponds to authentic $\mathrm{BM}^{53,54}$. In our system, cells and ECM were assembled in 3D on a microporous membrane that served merely as a support for the printing process unlike previously developed $3 \mathrm{D}$ in vitro models of the alveolar barrier where the membrane was used to mimic the basement membrane $e^{14,15,52}$, with different cell types cultured on both sides of a microporous membrane, which is foreign (non-biomimetic) and considerably thick restricting direct cell-cell interactions and thus limiting their biological relevance. In the here presented in vitro model we employed cell lines that have the inherent advantage of being homogeneous and more stable in comparison to primary human epithelial and endothelial lung cells ${ }^{16}$ and, hence, ensuring better control and reproducibility when performing screening tests. Such an approach will also allow an interlaboratory comparison. Nevertheless, in a further step we will evaluate the possibility to print primary cells, to reflect even a more realistic in vivo situation.

The integration of a dynamic system simulating breathing motions into models reconstituting the air-blood barrier has been proven by previous research ${ }^{20-22,24}$ to be crucial for recapitulating the in vivo situation at the air-liquid interface adding them an unprecedented value in terms of tissue functionality. We therefore aim to explore in the future the possibilities of combining cell printing with mechanically active microfluidic systems. Chang and co-authors have previously shown that a combinatorial setup of a 3D cell printing process and a microfluidic environment enabled the creation of an improved higher fidelity in vitro micro-liver tissue analogue, which might be useful in drug and toxicology screening ${ }^{50}$.

Morphological analysis revealed considerable differences between the two seeding approaches, both with respect to mono and co-cultures: conventional manual seeding resulted in the formation of multi-layered often discrete cell clusters which were embedded in thick Matrigel $^{\mathrm{TM}}$ layers (Fig. 3) with a thickness of about 20-30 $\mu \mathrm{m}$ that may have significant impact on direct cell-cell interactions. In comparison, by optimizing the printing process, very thin layers of Matrigel $^{\mathrm{TM}}$ (about $1-2 \mu \mathrm{m}$ ) could be obtained inducing a more optimal growth of the epithelial and endothelial cells, i.e. are structurally in a very close interaction, exhibiting a thin, outstretched morphology similar to physiological conditions that promote cell functionality. In the former case, the dispersion of a very thin ECM layer is limited by the fact that this hydrogel starts to form a gel at above $10^{\circ} \mathrm{C}$, a process which cannot be avoided by manual pipetting. On the other hand, the bioprinter dispensing system is equipped with a temperature controlled unit that ensures a constant temperature for thermo-sensitive biomaterials during the printing procedure. 
$8 \mathrm{~h}$
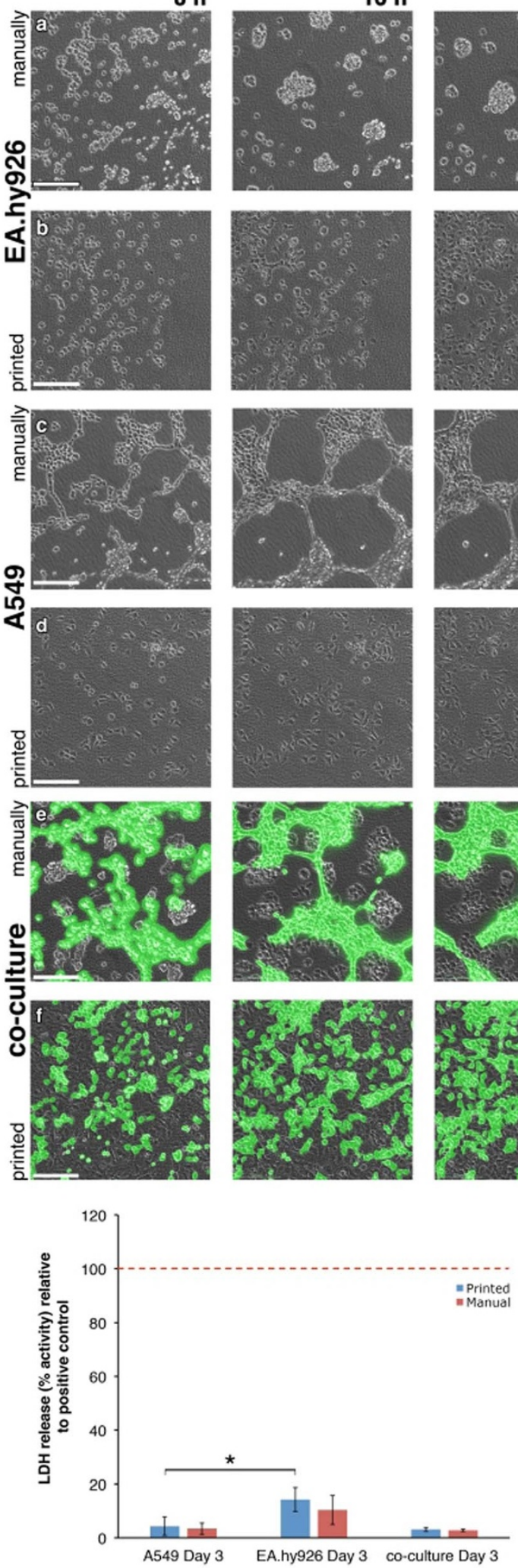

$24 \mathrm{~h}$
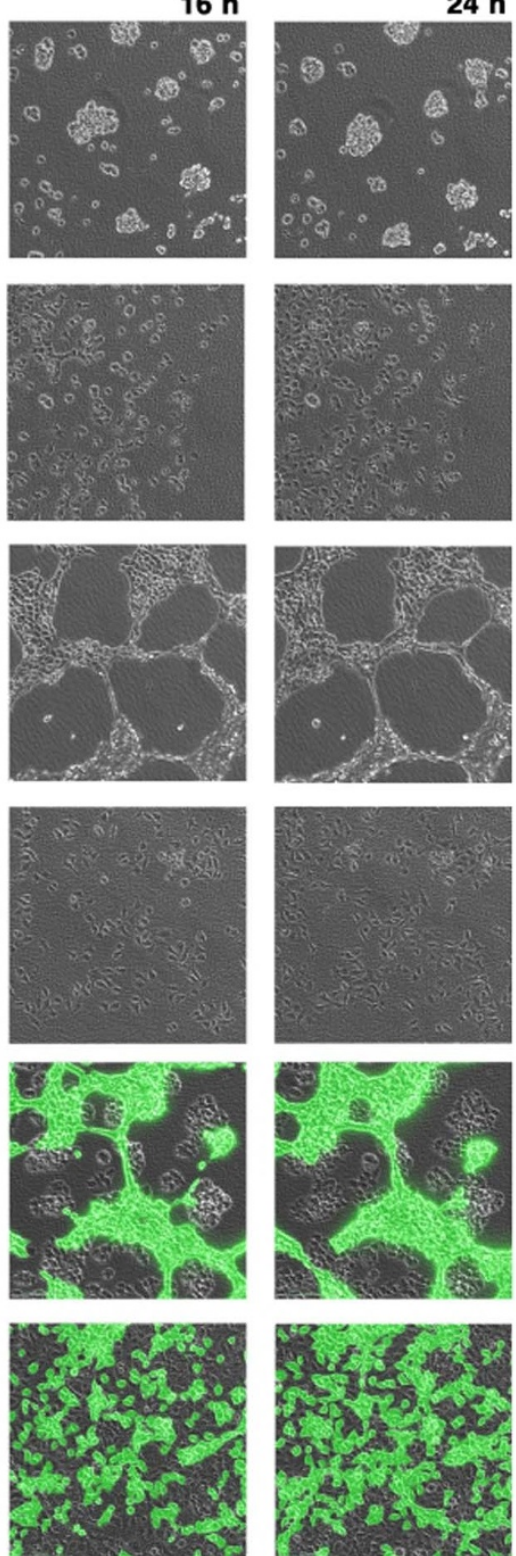

g
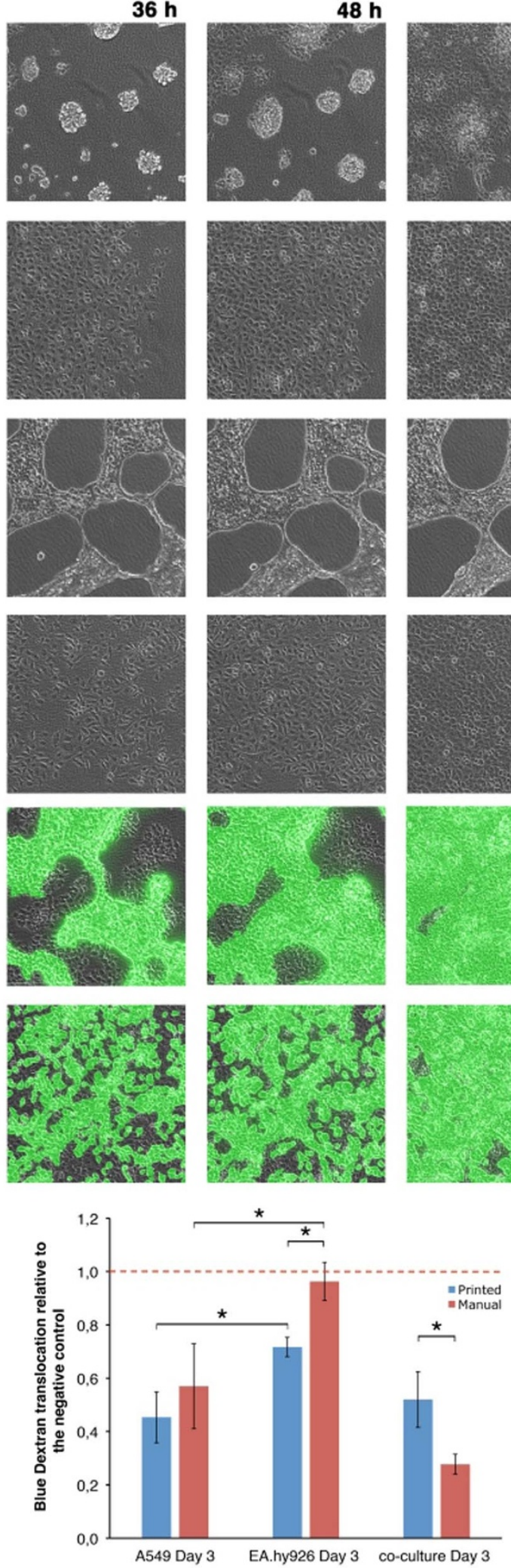

$72 \mathrm{~h}$
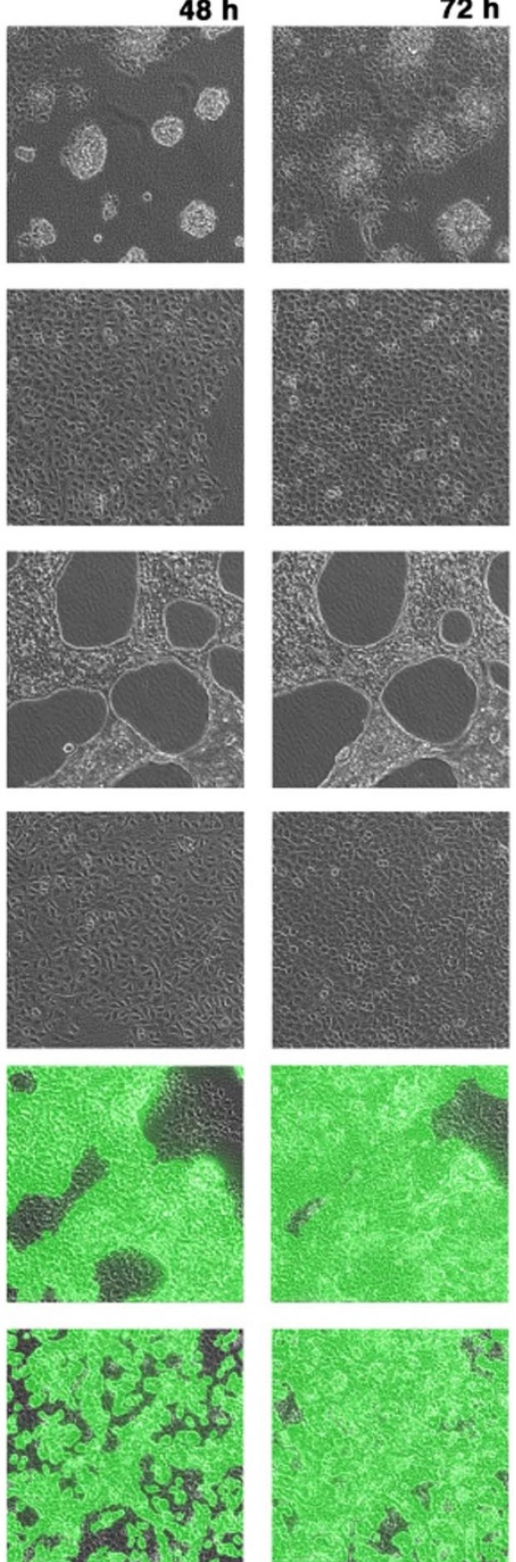

h

Figure 4 Qualitative analysis of cell proliferation and evaluation of cell viability and tightness of mono- and co-cultures assembled by two different approaches. Representative photomicrographs of manually seeded (a) EA.hy926, (c) A549 mono-cultures, (e) EA.hy926-A549 co-culture and printed cells: (b) EA.hy926, (d) A549 and (f) co-culture over 72 h in culture (Figs a-d are phase-contrast micrographs; Figs e-f are merged images of phase contrast and fluorescence micrographs). Scale bars correspond to $200 \mu \mathrm{m}$. (g) Cell integrity was estimated by quantification of extracellular LDH release (percent increase) relative to the positive control (Triton X-100 for $24 \mathrm{~h}$; red dashed line) 3 days after printing ( $\square$ ) or manual seeding ( $\square$ ) of cells on Matrigel $^{\mathrm{TM}}$. (h) Translocation of Blue Dextran was assessed on the $3^{\text {rd }}$ day of mono- and co-culture assembly on printed ( $\square$ ) and manually seeded ( $\square$ ) cultures. The values are shown as fold increase related to the negative control (insert only, w/o cells). All results are expressed as the mean \pm standard deviation of $n=3$ independent experiments $\left({ }^{*} p<0.05\right)$. 
We further detected high cell viability ( $>95 \%$ for epithelial cells and co-cultures and $\geq 86 \%$ for endothelial cells) and proliferation of the investigated cell types, both in mono- and co-cultures, which was essentially similar regardless of the employed approach (manual or printing). In this regard, we demonstrate that the valve-based printing which is considered to be the gentlest among the existing techniques for cell printing ${ }^{51}$, did not affect the majority of cells as evidenced by LDH-release measurements and by qualitative analysis of cell proliferation (Fig. 4a-g and Supplemetary Fig. S2). Moreover, printed epithelial and endothelial mono-cultures exhibited tighter layers than the corresponding manual cultures due to the fact that manually seeded cells organize in clusters resulting in patchy nonuniform cell layers, while printed cells have more uniform outstretched morphology (Fig. 2, 3). The observed higher permeability of endothelial cells (Fig. 4h) can also be explained with their biological function, i.e. the relatively leaky occluding junctions between the cells in vivo allow for nearly unlimited exchange of water, solutes and smaller macromolecules between the blood plasma and the interstitial space ${ }^{55}$. Similar observation, i.e. high permeability of EA.hy 926 cells has already been reported by Klein et al. ${ }^{15}$. The tightness of co-cultures produced by using two approaches was similar taking into account the barrier function of thick ECM layers that were present in manually established co-cultures.

The lack of a highly reliable and commercially available $3 \mathrm{D}$ in vitro alveolar model represents a major bottleneck for the development of efficacious and safe medicines across many therapeutic areas and risk assessment for inhaled xenobiotics or nanoparticles, such as environmental particulates or combustion-derived particles. In this project we communicate results on generating $3 \mathrm{D}$ alveolar tissue with a bioprinting approach, which is the first step towards the validation of printed lung tissue. In the near future, this novel approach will allow us to provide a realistic, automatized and easy to handle in vitro model for hazard assessment in the field of regulatory toxicology as well as for the development of new inhalative medicine.

\section{Methods}

Bioprinting platform and control software. The BioFactory ${ }^{\circledR}$ (regenHU Ltd. Switzerland) is a three-dimensional (3D) bioprinter with a printing resolution in the micrometre range $(<5 \mu \mathrm{m})$ and smallest printable volumes in the range of 5-10 nL. The platform consists of a desktop instrument enclosed within a sterile hood and a human machine interface (HMI) control software. The main component of the instrument is the process unit comprising (i) the tool changer with three workstations (upgradable to six workstations) equipped with print heads that allows for printing up to 3 different biomaterials/cells and (ii) the building cell with a micrometre-resolution three-axis building platform for biofabrication of $3 \mathrm{D}$ objects. The dispensing system consists of a CF $300 \mathrm{~N}$ valve-based print head, which can be used for jetting or contact dispensing with additionally mounted needle tip. The workstation used for ECM patterning was equipped with a temperature control unit that ensures a constant temperature along the whole dispensing system. ECM was printed by contact dispensing, while cells were printed with jetting. The material flow is controlled by an exchangeable micro valve that is mounted in the print head support housing the solenoid that generates the power to open the valve. The ECM/cells suspension is filled in cartridges, which are interconnected with print heads through a Luer-Lock thread adapter and an inlet adapter. The dispensing unit requires air pressure for the biomaterials to be dispensed from cartridges, which is supplied from a central inlet equipped with an air filter. The air is spread through air spouts to each station where the desired pressures are adjusted by individual pressure regulators. The hardware is controlled via the HMI control software. For designing patterns/scaffolds we used BioCAD (version 1.0), a 3D drawing suite developed by regenHU Ltd. From the pattern information defined in BioCAD a g-code file was created, loaded to HMI and launched after adjusting the printing parameters summarized in Supplementary Table S1.

Array printing. An experiment was carried out to test the positional repeatability and accuracy of the BioFactory ${ }^{\circledR}$, i.e., whether the precision of the manipulator is high enough to return to previous positions defined by specific coordinates without drifting in $\mathrm{x}$ or $\mathrm{y}$ direction. The models created for the control of the accuracy were a multi-layered circular grid scaffold and the logo of Adolphe Merkle Institute representing a more complex pattern, both printed from Nivea cream (Supplementary Fig. S4a-b). The former model was a circular (10 $\mathrm{mm}$ in diameter) scaffold with a height of $3 \mathrm{~mm}$ filled with coloured cream in the pores. It was made of alternating layers with vertical and horizontal lines with a porosity of about $60 \%$. Between each layer, the space between the lines was filled with droplets of Nivea cream coloured with red food dye (both can be found at Migros AG) to show that multiple print head can be used on the same construct with a high repeatability and space accuracy.

Cell culture. Experiments were carried out with the human alveolar epithelial type II cell line $\mathrm{A} 549^{56}$, and the EA.hy926 hybrid human cell line derived by fusing human umbilical vein endothelial cells (HUV-EC) with A549 cells ${ }^{57}$. Both cell lines were obtained from the American Type Culture Collection (ATCC, USA). For co-culture experiments A549 cells stably expressing the eGFP protein were used, which were kindly provided by Dr. B. Schwaller, Department of Medicine, University of Fribourg. The cells were cultured in the following media: A549 cells were maintained in RPMI 1640 (Gibco, Life Technologies Europe B.V., Zug, Switzerland); EA.hy926 cells were cultured in DMEM medium (Gibco) and the co-culture of the two cell types was cultivated in 1:1 RPMI 1640: DMEM medium. The cell culture media were supplemented with $10 \%(\mathrm{v} / \mathrm{v})$ fetal bovine serum (FBS; PAA Laboratories, Chemie Brunschwig AG, Basel, Switzerland), 1\% (v/v) L-Glutamine (Life Technologies Europe) and $1 \%(\mathrm{v} / \mathrm{v})$ penicillin/streptomycin (Gibco). The cells were maintained in a humidified incubator $\left(37^{\circ} \mathrm{C}, 5 \% \mathrm{CO}_{2}\right)$, medium was changed every $2-3$ days and the cells were subcultured through trypsinization when reached near-confluence. Initial cell concentrations were calculated using Trypan blue exclusion method $(0.4 \%$ Trypan blue solution, T8154; Sigma Aldrich Chemie GmbH, Buchs, Switzerland). The working cell concentrations were prepared by diluting cells with cell culture medium. For the experiments, cell suspensions were prepared at a density of $4.5 \times 10^{6}$ cells $/ \mathrm{mL}$

Extracellular matrix substrate and cell preparation for bioprinting. BD Matrigel ${ }^{\mathrm{TM}}$ BM Matrix Growth Factor-Reduced (BD, 356230; concentration: $7.8 \mathrm{mg} / \mathrm{mL}$; subsequently referred to as "Matrigel ${ }^{\mathrm{TM}}$ ") was thawed overnight at $4{ }^{\circ} \mathrm{C}$ on ice and was kept cool on ice until further use. For the printing procedure, Matrigel ${ }^{\mathrm{TM}}$ was loaded in a sterile $3 \mathrm{cc}$ cartridge that was previously connected with a Luer-Lock adapter to a print head. The cartridge containing the ECM was constantly cooled with a minichiller (Huber Swiss Services GmbH, Möhlin, Switzerland) that maintained a constant cartridge temperature at $5^{\circ} \mathrm{C}$. A predefined circular pattern filled with horizontal lines designed in BioCAD software was loaded to the HMI interface of the printer and a thin layer of Matrige ${ }^{\mathrm{TM}}$ was printed with contact dispensing microvalve CF300 (MVC03-006; regenHU Ltd.; nozzle diameter $0.3 \mathrm{~mm}$ ) on Millicell ${ }^{\circledR}-\mathrm{CM}$ Organotypic tissue culture plate inserts (PICM ORG 50; Biopore $^{\mathrm{TM}} \mathrm{CM}$ hydrophilized PTFE membranes with a growth area of $4.2 \mathrm{~cm}^{2}$ and $0.4 \mu \mathrm{m}$ pores in diameter; Milllipore AG Zug, Switzerland) with printing parameters summarized in Supplementary Table S1. The cell culture inserts with a printed layer of ECM were incubated at $37^{\circ} \mathrm{C}$ for $30 \mathrm{~min}$. Next, EA.hy 926 or A549 cells suspended in cell culture medium (former in supplemented DMEM, latter in supplemented RPMI 1640) at a density of $4.5 \times 10^{6}$ cells $/ \mathrm{mL}$ were loaded in sterile $3 \mathrm{cc}$ cartridges and one layer of a repeated circular BioCAD pattern was printed with jetting microvalve CF300 (MVJ01-006; regenHU Ltd.; nozzle diameter $0.1 \mathrm{~mm}$ ) and parameters found in Supplementary Table S1. The printed cells were incubated in a humidified incubator $\left(37^{\circ} \mathrm{C}, 5 \% \mathrm{CO}_{2}\right)$ for $2 \mathrm{~h}$. Following cell adhesion, cells were grown overnight in BD Falcon ${ }^{\mathrm{TM}} 6$-well tissue culture plates under submerged conditions. On the first day of incubation, the cell culture media was changed. Co-cultures were printed with the same printing parameters as described for mono-cultures (Supplementary Table S1). The sequence of printing was the following: first, a layer of Matrigel ${ }^{\mathrm{TM}}$ was printed followed by a printed layer of EA.hy 926 endothelial cells, which were cultured for 2 days before printing the next layer of ECM and the last layer comprising A549 epithelial cells (Fig. 1c).

For manual procedure, Matrigel ${ }^{\mathrm{TM}}(80 \mu \mathrm{L})$ was dispersed with cell scraper over the surface of cell culture inserts. All disposables, such as pipette tips, cell scrapers were pre-cooled at $-20^{\circ} \mathrm{C}$ in order to prevent premature polymerization of $\mathrm{Matrigel}^{\mathrm{TM}}$. The pre-coated membranes were incubated at $37^{\circ} \mathrm{C}$ for $30 \mathrm{~min}$ for ECM gelation. $4.5 \times$ $10^{6}$ cells/mL EA.hy926 or A549 cells were seeded on Matrigel ${ }^{\mathrm{TM}}$-coated inserts according to the cell suspension volumes dispensed during the printing process. The initial calculated cell densities of EA.hy 926 and A549 cells on the cell culture transwell inserts for both conditions i.e., printing and manual seeding were $1.4 \times 10^{5}$ and $1.3 \times$ $10^{5}$ cells $/ \mathrm{cm}^{2}$, respectively.

Cell viability assays. a) Monitoring cell proliferation. The cell confluence was analysed with an integrated cell culture observation device, BioStation CT (Nikon Instruments, Zurich, Switzerland), consisting of a tissue culture incubator with builtin inverted microscope suited to capture $2 \times$ to $40 \times$ phase contrast and fluorescent images. Cells were printed/manually seeded on cell culture transwell inserts as described above and grown in 6-well tissue culture plates for few hours to allow for cell adhesion. Next, samples were placed in Nikon BioStationCT and a time-lapse experiment was conducted for $72 \mathrm{~h}$. Bright field and fluorescence (in case of cocultures) images were acquired every $2 \mathrm{~h}$ from $8 \mathrm{~h}$ on and confluence rates were displayed at 8 h,16 h, $24 \mathrm{~h}, 36 \mathrm{~h}, 48 \mathrm{~h}$ and $72 \mathrm{~h}$.

Lactate dehydrogenase assay. For the quantification of cell death and cell lysis in printed and manual samples, a colorimetric assay was employed, which is based on the measurement of lactate dehydrogenase (LDH) activity released from the cytosol of damaged cells. For that, cell culture supernatants were collected 3 days after printing or manual seeding and stored at $4{ }^{\circ} \mathrm{C}$ until analysis. The LDH cytotoxicity detection kit (Roche Applied Science, Mannheim, Germany) was used according to the supplier's manual. LDH was quantified photometrically by measuring at $490 \mathrm{~nm}$, 
with $630 \mathrm{~nm}$ as reference wavelength. Each sample was assessed in triplicate. For positive controls mono- and co-cultures were exposed to $0.5 \%$ Triton $\mathrm{X}-100$ detergent in $\mathrm{H}_{2} \mathrm{O}$ for $24 \mathrm{~h}$ at $37^{\circ} \mathrm{C}$.

Blue Dextran cell layer integrity assay. The tightness of mono- and co-cultures was investigated with Blue Dextran dye (Mw 2000 kDa; 17-0360-01 GE Healthcare, Sigma Aldrich). The principle of the assay is that when the Blue Dextran molecules are added to the apical side of intact epithelial cell monolayers grown on microporous membrane, the tight junctions between cells will prevent the paracellular transport of the dy ${ }^{58}$. Briefly, cells (printed or manually seeded) grown on Millicell ${ }^{\circledR}$ inserts for 3 days were rinsed with PBS, $1 \mathrm{~mL}$ of complete phenol red free cell culture medium was added in plate wells, in the lower compartment of the inserts and $250 \mu \mathrm{L}$ to the upper compartment on cell layers. Next, $250 \mu \mathrm{L}$ of $1 \%$ Blue Dextran (dissolved in PBS) was added to the apical surface and incubated for $2 \mathrm{~h}$ at $37^{\circ} \mathrm{C}$ in the incubator. The contents of lower and upper compartments were collected separately and the passage of Blue Dextran in basolateral samples was finally quantified using multimode plate reader (BioRad Benchmark Plus, R\&D Systems) by measuring the absorbance at $600 \mathrm{~nm}$.

Immunocytochemistry. The insert membranes with cells were washed in phosphate buffered saline (PBS) and fixed for $15 \mathrm{~min}$ at room temperature in $4 \%$ paraformaldehyde (PFA) in PBS. The fixed cells were incubated with primary and secondary antibodies for $2 \mathrm{~h}$ and $60 \mathrm{~min}$, respectively, at room temperature in dark. Antibodies were diluted in PBS (containing 0.3\% Triton X-100 and 1\% BSA) as follows: mouse anti-human PECAM-1 (10G9) 1:50 (sc-13537; Santa Cruz Biotechnology, Heidelberg, Germany), mouse anti-human VE-cadherin (F-8) $1: 50$ (sc-9989; Santa Cruz Biotechnology, Heidelberg, Germany), goat anti-mouse IgG H\&L 1 : 200 (Alexa Fluor ${ }^{\circledR} 647$ ab150119; Lucernachem, Luzern, Switzerland). The cytoskeleton i.e., F-actin-filaments of the cells were stained with rhodamine phalloidin 1:50 (R-415; Molecular Probes, Life Technologies Europe B.V., Zug, Switzerland) and cell nuclei were stained with DAPI $1: 100(1 \mu \mathrm{g} / \mathrm{mL}$; Sigma Aldrich). The samples were finally mounted in Dako Glycergel Mounting Medium (DAKO Schweiz AG, Baar, Switzerland).

Laser scanning microscopy and image restoration. An inverted Zeiss laser scanning microscope (LSM) 710 (Axio Observer.Z1, Zeiss, Switzerland) equipped with Ar $488 \mathrm{~nm}, \mathrm{HeNe} 543 \mathrm{~nm}$ and HeNe $633 \mathrm{~nm}$ lasers was used for visualization of samples and image acquisition. LSM images were further analysed using Imaris, a three-dimensional multi-channel image processing software (Bitplane AG, Zurich, Switzerland).

Histological staining and brightfield microscopy. To visualize and compare the presence and thickness of ECM layers in samples prepared with two approaches (printed vs. manual) they were stained with Masson Goldner trichrome stain suited for distinguishing cells from surrounding connective tissue. Briefly, samples were fixed in $4 \%$ PFA, dehydrated in $100 \%$ ethanol and xylol and subsequently embedded in paraffin. Serial $10-\mu \mathrm{m}$ thick paraffin sections were cut with a microtome and collected on Super Frost Plus slides. The staining was performed on rehydrated sections following standard procedures and resulted red cytoplasm, green collagen fibers and dark brown cell nuclei. Bright field images $(20 \times)$ were acquired with a Hamamatsu digital slide scanner (NanoZoomer 2.0-HT, Hamamatsu Photonics) and further analysed with NDP.view2 viewer software (Hamamatsu Photonics).

Statistical analysis. Results were averages from three independent experiments; each sample was assessed in triplicates. The results are presented as mean \pm SD. A one-way analysis of variance (ANOVA) was performed by using GraphPad Prism software (version 6; GraphPad Software Inc., La Jolla, California, USA) and considered significance at $p<0.05$.

1. Hartung, T. \& Rovida, C. Chemical regulators have overreached. Nature 460 , 1080-1081, doi:10.1038/4601080a (2009).

2. Andersen, M. E. Calling on Science: Making “Alternatives" the New Gold Standard. ALTEX 27, 135-143 (2010).

3. National Research Council Toxicity Testing in the 21st Century: A Vision and a Strategy. (National Academies Press, Washington, D.C., 2007).

4. Reduce, refine, replace. Nat. Immunol. 11, 971, doi:10.1038/ni1110-971 (2010).

5. Lee, C. J., Lee, L. H., Wu, C. L., Lee, B. R. \& Chen, M. L. Clinical Trials of Drugs and Biopharmaceuticals. (CRC Press, Taylor \& Francis Group, Boca Raton, 2005).

6. Jud, C., Clift, M. J., Petri-Fink, A. \& Rothen-Rutishauser, B. Nanomaterials and the human lung: what is known and what must be deciphered to realise their potential advantages? Swiss Med. Wkly. 143, w13758, doi:10.4414/smw.2013.13758 (2013).

7. de Souza Carvalho, C., Daum, N. \& Lehr, C. M. Carrier interactions with the biological barriers of the lung: Advanced in vitro models and challenges for pulmonary drug delivery. Adv. Drug Deliv. Rev. 75C, 129-140, doi:10.1016/ j.addr.2014.05.014 (2014).

8. Gehr, P. et al. Endocytosis of environmental and engineered micro- and nanosized particles. Compr. Physiol. 1, 1159-1174, doi:10.1002/cphy.c100035 (2011).

9. Peters, A., Wichmann, H. E., Tuch, T., Heinrich, J. \& Heyder, J. Respiratory effects are associated with the number of ultrafine particles. Am. J. Respir. Crit. Care Med. 155, 1376-1383, doi:10.1164/ajrccm.155.4.9105082 (1997).
10. Schulz, H. et al. Cardiovascular effects of fine and ultrafine particles. J. Aerosol Med. 18, 1-22, doi:10.1089/jam.2005.18.1 (2005).

11. Rothen-Rutishauser, B., Clift, M. J. D., Jud, C., Fink, A. \& Wick, P. Human epithelial cell in vitro - Are they an advantageous tool to help understand the nanomaterial-biological barrier interaction? Euro Nano Tox Letters 4, 1-20 (2012).

12. Fuchs, S., Gumbleton, M., Schaefer, U. F. \& Lehr, C. M. [Models of the alveolar epithelium] Cell Culture Models of Biological Barriers: In vitro Test Systems for Drug Absorption and Delivery [Lehr, C. M. (ed.)] [189-210] (Taylor \& Francis, London, New York, 2002).

13. Steimer, A., Haltner, E. \& Lehr, C. M. Cell culture models of the respiratory tract relevant to pulmonary drug delivery. J. Aerosol Med. 18, 137-182, doi:10.1089/ jam.2005.18.137 (2005)

14. Hermanns, M. I., Unger, R. E., Kehe, K., Peters, K. \& Kirkpatrick, C. J. Lung epithelial cell lines in coculture with human pulmonary microvascular endothelial cells: development of an alveolo-capillary barrier in vitro. Lab. Invest. 84, 736-752, doi:10.1038/labinvest.3700081 (2004).

15. Klein, S. G., Serchi, T., Hoffmann, L., Blomeke, B. \& Gutleb, A. C. An improved 3D tetraculture system mimicking the cellular organisation at the alveolar barrier to study the potential toxic effects of particles on the lung. Part. Fibre Toxicol. 10, 31, doi:10.1186/1743-8977-10-31 (2013).

16. Rothen-Rutishauser, B., Blank, F., Muhlfeld, C. \& Gehr, P. In vitro models of the human epithelial airway barrier to study the toxic potential of particulate matter. Expert Opin. Drug Metab. Toxicol. 4, 1075-1089, doi:10.1517/17425255.4.8.1075 (2008).

17. Nichols, J. E. et al. Modeling the lung: Design and development of tissue engineered macro- and micro-physiologic lung models for research use. Exp. Biol. Med. (Maywood) 239, 1135-1169, doi:10.1177/1535370214536679 (2014).

18. Gehr, P., Bachofen, M. \& Weibel, E. R. The normal human lung: ultrastructure and morphometric estimation of diffusion capacity. Respir. Physiol. 32, 121-140 (1978).

19. Weibel, E. R. What makes a good lung? Swiss Med. Wkly. 139, 375-386, doi:smw12270 (2009)

20. Huh, D. et al. Reconstituting organ-level lung functions on a chip. Science 328, 1662-1668, doi:10.1126/science.1188302 (2010)

21. Huh, D. et al. A human disease model of drug toxicity-induced pulmonary edema in a lung-on-a-chip microdevice. Sci. Transl. Med. 4, 159ra147, doi:10.1126/ scitranslmed.3004249 (2012).

22. Huh, D. et al. Microfabrication of human organs-on-chips. Nat. Protoc. 8 , 2135-2157, doi:10.1038/nprot.2013.137 (2013).

23. Mahto, S. K. et al. Microfluidic platforms for advanced risk assessments of nanomaterials. Nanotoxicology, 1-15, doi:10.3109/17435390.2014.940402 (2014).

24. Schurch, D. et al. Modeling nanoparticle-alveolar epithelial cell interactions under breathing conditions using captive bubble surfactometry. Langmuir 30 4924-4932, doi:10.1021/la500307q (2014)

25. Mironov, V. et al. Biofabrication: a 21st century manufacturing paradigm. Biofabrication 1, 022001, doi:10.1088/1758-5082/1/2/022001 (2009).

26. Ozbolat, I. T. \& Yu, Y. Bioprinting toward organ fabrication: challenges and future trends. IEEE Trans. Biomed. Eng. 60, 691-699, doi:10.1109/TBME.2013.2243912 (2013).

27. Tasoglu, S. \& Demirci, U. Bioprinting for stem cell research. Trends Biotechnol. 31, 10-19, doi:10.1016/j.tibtech.2012.10.005 (2013).

28. Wust, S., Godla, M. E., Muller, R. \& Hofmann, S. Tunable hydrogel composite with two-step processing in combination with innovative hardware upgrade for cell-based three-dimensional bioprinting. Acta Biomater. 10, 630-640, doi:10.1016/j.actbio.2013.10.016 (2014).

29. Guillotin, B. \& Guillemot, F. Cell patterning technologies for organotypic tissue fabrication. Trends Biotechnol. 29, 183-190, doi:10.1016/j.tibtech.2010.12.008 (2011).

30. Murphy, S. V. \& Atala, A. 3D bioprinting of tissues and organs. Nat. Biotechnol. 32, 773-785, doi:10.1038/nbt.2958 (2014).

31. Bajaj, P., Schweller, R. M., Khademhosseini, A., West, J. L. \& Bashir, R. 3D biofabrication strategies for tissue engineering and regenerative medicine. Annu. Rev. Biomed. Eng. 16, 247-276, doi:10.1146/annurev-bioeng-071813-105155 (2014).

32. Seol, Y. J., Kang, H. W., Lee, S. J., Atala, A. \& Yoo, J. J. Bioprinting technology and its applications. Eur. J. Cardiothorac. Surg. 46, 342-348, doi:10.1093/ejcts/ezu148 (2014).

33. Duan, B., Kapetanovic, E., Hockaday, L. A. \& Butcher, J. T. Three-dimensional printed trileaflet valve conduits using biological hydrogels and human valve interstitial cells. Acta Biomater. 10, 1836-1846, doi:10.1016/j.actbio.2013.12.005 (2014).

34. Gaebel, R. et al. Patterning human stem cells and endothelial cells with laser printing for cardiac regeneration. Biomaterials 32, 9218-9230, doi:10.1016/ j.biomaterials.2011.08.071 (2011).

35. Gaetani, R. et al. Cardiac tissue engineering using tissue printing technology and human cardiac progenitor cells. Biomaterials 33, 1782-1790, doi:10.1016/ j.biomaterials.2011.11.003 (2012).

36. Pati, F. et al. Printing three-dimensional tissue analogues with decellularized extracellular matrix bioink. Nat. Commun. 5, 3935, doi:10.1038/ncomms 4935 (2014). 
37. Fedorovich, N. E., De Wijn, J. R., Verbout, A. J., Alblas, J. \& Dhert, W. J. Threedimensional fiber deposition of cell-laden, viable, patterned constructs for bone tissue printing. Tissue Eng. Part A 14, 127-133, doi:10.1089/ten.a.2007.0158 (2008).

38. Fedorovich, N. E. et al. Biofabrication of osteochondral tissue equivalents by printing topologically defined, cell-laden hydrogel scaffolds. Tissue Eng. Part C, Methods 18, 33-44, doi:10.1089/ten.TEC.2011.0060 (2012).

39. Phillippi, J. A. et al. Microenvironments engineered by inkjet bioprinting spatially direct adult stem cells toward muscle- and bone-like subpopulations. Stem Cells 26, 127-134, doi:10.1634/stemcells.2007-0520 (2008).

40. Xu, T. et al. Hybrid printing of mechanically and biologically improved constructs for cartilage tissue engineering applications. Biofabrication 5, 015001, doi:10.1088/1758-5082/5/1/015001 (2013)

41. Cui, X. \& Boland, T. Human microvasculature fabrication using thermal inkjet printing technology. Biomaterials 30, 6221-6227, doi:10.1016/ j.biomaterials.2009.07.056 (2009).

42. Norotte, C., Marga, F. S., Niklason, L. E. \& Forgacs, G. Scaffold-free vascular tissue engineering using bioprinting. Biomaterials 30, 5910-5917, doi:10.1016/ j.biomaterials.2009.06.034 (2009)

43. Bertassoni, L. E. et al. Hydrogel bioprinted microchannel networks for vascularization of tissue engineering constructs. Lab Chip 14, 2202-2211, doi:10.1039/c4lc00030g (2014).

44. Lee, W. et al. Multi-layered culture of human skin fibroblasts and keratinocytes through three-dimensional freeform fabrication. Biomaterials 30, 1587-1595, doi:10.1016/j.biomaterials.2008.12.009 (2009)

45. Marga, F. et al. Toward engineering functional organ modules by additive manufacturing. Biofabrication 4, 022001, doi:10.1088/1758-5082/4/2/022001 (2012).

46. Owens, C. M., Marga, F., Forgacs, G. \& Heesch, C. M. Biofabrication and testing of a fully cellular nerve graft. Biofabrication 5, 045007, doi:10.1088/1758-5082/5/4/ 045007 (2013).

47. Xu, F. et al. A three-dimensional in vitro ovarian cancer coculture model using a high-throughput cell patterning platform. Biotechnol. J. 6, 204-212, doi:10.1002/ biot.201000340 (2011).

48. Zhao, Y. et al. Three-dimensional printing of Hela cells for cervical tumor model in vitro. Biofabrication 6, 035001, doi:10.1088/1758-5082/6/3/035001 (2014).

49. Chang, R., Nam, J. \& Sun, W. Direct cell writing of 3D microorgan for in vitro pharmacokinetic model. Tissue Eng. Part C, Methods 14, 157-166, doi:10.1089/ ten.tec.2007.0392 (2008).

50. Chang, R., Emami, K., Wu, H. \& Sun, W. Biofabrication of a three-dimensional liver micro-organ as an in vitro drug metabolism model. Biofabrication 2, 045004 doi:10.1088/1758-5082/2/4/045004 (2010).

51. Faulkner-Jones, A. et al. Development of a valve-based cell printer for the formation of human embryonic stem cell spheroid aggregates. Biofabrication 5, 015013, doi:10.1088/1758-5082/5/1/015013 (2013).

52. Rothen-Rutishauser, B. M., Kiama, S. G. \& Gehr, P. A three-dimensional cellular model of the human respiratory tract to study the interaction with particles. Am. J. Respir. Cell Mol. Biol. 32, 281-289, doi:10.1165/rcmb.2004-0187OC (2005).

53. Benton, G., Arnaoutova, I., George, J., Kleinman, H. K. \& Koblinski, J. Matrigel: From discovery and ECM mimicry to assays and models for cancer research. Adv. Drug Deliv. Rev. doi:10.1016/j.addr.2014.06.005 (2014).
54. Hughes, C. S., Postovit, L. M. \& Lajoie, G. A. Matrigel: a complex protein mixture required for optimal growth of cell culture. Proteomics 10, 1886-1890, doi:10.1002/pmic.200900758 (2010).

55. Fishman, A. P. et al. Fishman's Pulmonary Diseases and Disorders (The McGrawHill Companies, New York, 2008).

56. Lieber, M., Smith, B., Szakal, A., Nelson-Rees, W. \& Todaro, G. A continuous tumor-cell line from a human lung carcinoma with properties of type II alveolar epithelial cells. Int. J. Cancer 17, 62-70 (1976).

57. Edgell, C. J., McDonald, C. C. \& Graham, J. B. Permanent cell line expressing human factor VIII-related antigen established by hybridization. Proc. Natl. Acad. Sci. U. S. A. 80, 3734-3737 (1983)

58. Velarde, G. et al. Use of transepithelial electrical resistance in the study of pentachlorophenol toxicity. Toxicol. In Vitro 13, 723-727 (1999).

\section{Acknowledgments}

We thank M. Sanchez for her great experimental assistance during histological sample preparation, C. Wotzkow for his excellent technical support during live cell imaging experiments. The authors acknowledge the expert technical assistance of $\mathrm{M}$. Thurner, $\mathrm{M}$. Kuster, R. Wenger and A. Scheidegger. This work was supported by the Innovation Fund Fribourg, the Adolphe Merkle Foundation, the Research Fund of the Swiss Lung Association, Bern and the Novartis Research Foundation.

\section{Author contributions}

The study was conceived and designed by L.H. and B.R.R. C.J. performed preliminary experiments. L.H. and Y.U. performed cell viability-, cell layer integrity experiments and immunocytochemistry. L.H. and F.B. performed time-lapse experiments for monitoring cell proliferation. L.H. performed all the other experiments. L.H. and B.R.R. analysed the data and wrote the manuscript. The study was directed by B.R.R. and A.P.F. All authors discussed the results and commented on the manuscript.

\section{Additional information}

Supplementary information accompanies this paper at http://www.nature.com/ scientificreports

Competing financial interests: The authors declare no competing financial interests. The acquisition of the Bioprinter was supported by the Innovationfond Fribourg by a proposal of Prof. B. Rothen-Rutishauser and Marc Thurner, the owner of regenHU Ltd.

How to cite this article: Horváth, L. et al. Engineering an in vitro air-blood barrier by 3D bioprinting. Sci. Rep. 5, 7974; DOI:10.1038/srep07974 (2015).

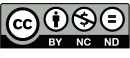

This work is licensed under a Creative Commons Attribution-NonCommercialNoDerivs 4.0 International License. The images or other third party material in this article are included in the article's Creative Commons license, unless indicated otherwise in the credit line; if the material is not included under the Creative Commons license, users will need to obtain permission from the license holder in order to reproduce the material. To view a copy of this license, visit http:// creativecommons.org/licenses/by-nc-nd/4.0/ 